\title{
Sex Differences in Physical Activity in People After Stroke: A Cross-sectional Study
}

\author{
Wataru NAKANO ${ }^{1}$, Satomi KobaYAShi, PT, $\mathrm{PhD}^{2}$, Takayuki MaEZAWA, $\mathrm{PT}^{3}$, \\ Yukari OHASHI, PT, $\mathrm{PhD}^{4}$ and Yutaka KoHNO, MD, $\mathrm{PhD}^{5}$ \\ ${ }^{1)}$ Department of Physical Therapy, Tokoha University, Japan \\ ${ }^{2)}$ Department of Physical Therapy, Tsukuba International University, Japan \\ ${ }^{3)}$ Department of Physical Therapy, Ibaraki Prefectural University of Health Sciences Hospital, Japan \\ ${ }^{4)}$ Department of Physical Therapy, Ibaraki Prefectural University of Health Sciences, Japan \\ ${ }^{5)}$ Department of Neurology, Ibaraki Prefectural University of Health Sciences Hospital, Japan
}

\begin{abstract}
OBJECTIVE: Adequate physical activity after stroke is critical for cardiovascular health. Although sex is a potential factor associated with post-stroke physical activity, its mechanism remains unclear. This study aimed to examine sex differences in human physical activity following stroke. METHOD: A cross-sectional study with 62 participants (men: 42, women: 20) was conducted. Physical activity was measured for three consecutive days using a step activity monitor. The walking durations per day in light physical activity, moderate-to-vigorous physical activity, and total physical activity were calculated. Sex differences in walking duration were compared using Welch's t-tests or Mann-Whitney U tests. RESULTS: Women had a significantly greater walking duration in light physical activity and in total than did the men. In contrast, no significant differences were found in moderate-to-vigorous physical activity. CONCLUSION: This study reported sex differences in the walking duration after stroke. Moreover, it found that women spent more time in low intensity physical activity than men. Our results will be useful for planning interventions to increase physical activity and decrease sedentary behavior after stroke.
\end{abstract}

Key words: Physical activity, Stroke, Intensity, Guideline

(Phys Ther Res 00: 00-00, 0000)

$\mathbf{I}_{\text {ncreasing physical activity (PA) after stroke is important }}$ to control risk factors associated with further cardiovascular disease $^{1)}$. The American Stroke and Heart Association's PA recommendations ${ }^{2)}$ state that people with stroke should engage in aerobic activity, defined as $40-70 \%$ of the maximal oxygen consumption reserve or heart rate reserve (equivalent to moderate-vigorous physical activity; MVPA) for 2060 minutes, 3-5 days per week. Nevertheless, many community-dwelling people with stroke are physically in$\operatorname{active}^{3)}$.

Although sex is a potential factor associated with post-

Received: February 2, 2021

Accepted: April 7, 2021

Advance Publication by J-STAGE: July 13, 2021

Correspondence to: Wataru Nakano, Department of Physical Therapy, Tokoha University, Japan, 1-30 Mizuochicho, Aoi-ku, Shizuokashi, Shizuoka-ken 420-0831, Japan

\# e-mail:w-nakano@sz.tokoha-u.ac.jp

doi: 10.1298/ptr.E10099 stroke PA, the results obtained to date are controversial ${ }^{4-6)}$. Two systematic reviews ${ }^{4,5)}$ concluded that there were no significant correlations between PA levels and sex. Another meta-analysis $^{6)}$ reported a small relationship between PA and sex. However, the meta-analysis findings ${ }^{6)}$ were based on the results of only two studies $(n=58)$.

Sex may have an impact on different PA categories ${ }^{7,8)}$. In healthy older populations, men tend to spend more time in some domains, including vigorous and leisure-time PA, compared with women, whereas women tend to spend more time in light physical activity (LPA), such as in household and non-leisure/non-sport activities compared with that in men ${ }^{7,8)}$. In terms of MVPA patterns, one study ${ }^{9)}$ reported that women accumulated more short bouts (1-9 minutes) of MVPA than men did; in contrast, men accumulated more long bouts ( $>10$ minutes) of MVPA than women did. Thus, it is necessary to consider the PA intensity and MVPA bout length when analyzing sex differences in PA after stroke.

The present study aimed to examine the sex differ- 
ences in physical activity after stroke. We hypothesized that women would spend more time than men in LPA and shortbout MVPA, and that men would spend more time than women in long-bout MVPA.

\section{Methods}

\section{Study design and participants}

This study used a cross-sectional design and is part of a prospective study aiming to determine the longitudinal PA changes in stroke people after discharge. Individuals with ischemic or hemorrhagic stroke were recruited from a rehabilitation hospital. All participants were enrolled at discharge from the rehabilitation ward. Inclusion criteria were 1) first-ever stroke, 2) discharge to home, 3) independently mobile over 10 meters with or without walking aids or orthosis, and 4) able to understand simple instructions. Exclusion criteria were 1) Mini-Mental State Examination score $<24,2$ ) mobility problems before stroke, and 3) severe medical conditions, such as recent myocardial infarction or unstable angina. All individuals provided written informed consent to participate. This study was approved by the Ethics Committee at Ibaraki Prefectural University of Health Sciences (approval number: e171).

\section{Procedure}

Measurements of participant characteristics were collected at discharge and home visit. A systematic review ${ }^{6}$ reported that age, sex, physical function, depression, fatigue, and self-efficacy were factors associated with post-stroke physical activity. The 2-minute walk test ${ }^{10)}$ (one trial), 10meter walk test ${ }^{11)}$ (three trials at fastest speed), timed-up and go test ${ }^{12)}$ (three trials) were performed at discharge to measure physical function. The Mini-Mental State Examination $^{13)}$ (cognitive status) was also examined. Then, research staff conducted a home visit within one month after discharge and evaluated patients via the Patient Health Questionnaire-9 ${ }^{14)}$ (depression), Fatigue Severity Scale ${ }^{15}$ (fatigue), Modified Falls Efficacy Scale ${ }^{16)}$ (self-efficacy) and activity monitor (PA).

A StepWatch Activity Monitor (Modus Health, Edmonds, Washington) was strapped above the lateral malleolus of the less-affected lower extremity. The StepWatch Activity Monitor is a valid and reliable tool for measuring walking activity in people with stroke ${ }^{17)}$. Participants were asked to wear the monitor for three consecutive days during all waking hours, except during bathing and swimming activities. At discharge, participants received standard advice to perform PA, but no instruction was given to encourage PA at home visit. Step data were collected every 5 seconds.

\section{Data Analysis}

Data for wearing times greater than 8 hours per day were used for data analysis. Non-wearing was defined when no stride was detected for at least 90 consecutive minutes. The start of a walking bout was defined as three strides within a 15-second interval, and the end of a walking bout was defined as a 10-second interval in which no strides occurred $^{18,19)}$. Because the StepWatch Activity Monitor detects a stride on the mounting side, the output was doubled and expressed as steps. The number of steps, walking duration, and walking cadence for each walking bout were calculated.

To define the intensity of activity, we calculated the peak cadence ratio as the ratio of walking cadence in each walking bout to the maximum walking cadence during the measurement period. For example, when the walking cadence in a walking bout was 50 steps $/ \mathrm{min}$ and the maximum walking cadence during the measurement period was $100 \mathrm{steps} / \mathrm{min}$, the peak cadence ratio was 0.5. Energy expenditure in walking at a comfortable pace among stroke survivors was approximately 3 metabolic equivalents ${ }^{20)}$, corresponding to MVPA. Cadence during low-sequentialstep bouts cannot be considered a comfortable walking pace, because these activities are performed at a slower speed $^{21)}$. In contrast, cadence during high-sequential-step bouts can be used to define MVPA because it represents walking toward a destination or exercise ${ }^{22)}$. We operationally defined a high-sequential-step bout as $\geq 300$ steps $^{19)}$ and classified each walking bout into either LPA or MVPA based on the peak cadence ratio during high-sequential-step bouts.

We used SPSS version 25 (IBM Corp, Armonk, NY) for the statistical analyses. Sex differences in participant characteristics were compared using Fisher's exact test, Welch's t-tests, or Mann-Whitney U tests according to the normality of the data. We used the cut-off point of a highsequential-step bout ( $\geq 300$ steps) in relation to the peak cadence ratio to define activity intensity (LPA or MVPA). The cut-off point was determined by receiver operating characteristic curves and Youden's index. Then, walking durations per day in LPA, MVPA, and total (LPA plus MVPA) were calculated. MVPA was further divided into two types according to length of each walking bout: shortbout MVPA (1-9 min) and long-bout MVPA ( $\geq 10 \mathrm{~min})^{9)}$. The walking durations per day are expressed as the product of duration per bout and the number of bouts per day. Therefore, we calculated the median duration per bout and the number of bouts per day in LPA, MVPA, and Total. The step activity was calculated for each day, and the average values across days were used in the analysis of group differences. Sex differences in each walking variable were compared using Welch's t-tests or Mann-Whitney U tests according to the normality of the data. A p-value $<0.05$ was used to determine statistical significance. 
Table 1. Participant demographics

\begin{tabular}{lccc}
\hline & Men $(\mathrm{n}=42)$ & Women $(\mathrm{n}=20)$ & $p$-value \\
\hline Age (y) & $63 \pm 13$ & $64 \pm 7$ & 0.89 \\
Stroke type, n (infarction/hemorrhage) & $29 / 13$ & $10 / 10$ & 0.12 \\
Lesion side, n (right/left) & $27 / 15$ & $3 / 17$ & $<0.001$ \\
Post-stroke duration (days) & $143 \pm 51$ & $155 \pm 49$ & 0.41 \\
Post-discharge duration (days) & $17 \pm 9$ & $16 \pm 8$ & 0.66 \\
Mini-Mental State Examination (0-30) & $30(26-30)$ & $29(25-30)$ & 0.11 \\
Patient Health Questionnaire-9 (0-27) & $2(0-5)$ & $1(0-5)$ & 0.79 \\
Fatigue Severity Scale (9-63) & $24(16-40)$ & $18(14-30)$ & 0.29 \\
Modified Falls Efficacy Scale (0-140) & $129(105-139)$ & $131(119-139)$ & 0.44 \\
Walking with orthosis, $\mathrm{n}(\%)$ & $16(38 \%)$ & $5(25 \%)$ & 0.23 \\
Walking with cane, $\mathrm{n}(\%)$ & $18(43 \%)$ & $8(40 \%)$ & 0.53 \\
2MWD (m) & $150 \pm 45 \dagger$ & $153 \pm 47$ & 0.79 \\
Gait speed: fastest (m/s) & $1.5 \pm 0.7 \dagger$ & $1.5 \pm 0.5$ & 0.82 \\
Timed up and go test (s) & $12(9-14) \neq$ & $10(8-14)$ & 0.26 \\
\hline
\end{tabular}

Abbreviations: 2MWD, 2-minute walk distance

$\dagger \mathrm{n}=1$ missing, $\ddagger \mathrm{n}=2$ missing

Mini-Mental State Examination score ranges from 0 to 30. High scores indicate good cognitive function. Patient Health Questionnaire-9 score ranges from 0 to 27 . High scores indicate higher levels of depressive symptoms. The Fatigue Severity Scale score ranges from 9 to 63. High scores indicate greater fatigue severity. The Modified Falls Efficacy Scale score ranges from 0 to 140 . High scores indicate higher fall efficacy.

\section{Results}

In total, 239 people with stroke were screened, 162 fulfilled the inclusion criteria and 62 participants (42 men, 20 women) agreed to participate in the study. Table 1 presents the participant characteristics and the comparisons between men and women. No sex differences were found among factors associated with post-stroke $\mathrm{PA}^{6)}$. Although PA data for 10 participants (men: seven; women: three) were available for only two days, all 62 participants were included in the analyses. Mean wearing time was $712 \pm$ $144 \mathrm{~min} /$ day in men and $803 \pm 122 \mathrm{~min} /$ day in women.

The receiver operating characteristic curves analysis revealed that the area under the curve for high-sequentialstep bout ( $\geq 300$ steps) in relation to the peak cadence ratio was 0.961 (95\% confidence interval: 0.957-0.966), and the optimal cut-off value was 0.655 , correlating to $65.5 \%$ of the maximum walking cadence during the measurement period. Therefore, walking bouts in which the peak cadence ratio was less than 0.655 were defined as LPA, and those with a peak cadence ratio greater than or equal to 0.655 were defined as MVPA. The mean maximum walking cadence during the measurement period for all participants was $106 \pm$ $14 \mathrm{steps} / \mathrm{min}$. The cut-off value corresponded to a walking cadence of $69 \pm 9$ steps/min.

Table 2 presents the comparison of sex differences in walking duration. Women had a significantly greater walking duration in total and LPA. No sex differences were found in any parameter in MVPA. In all participants, the average MVPA duration was 44 minutes. Of these, 75\% were short-bout MVPA. Figure 1 shows a comparison of sex differences in the median duration per bout and the number of bouts per day. Women had a significantly greater number of Total and LPA bouts per day. No sex differences were found in the median duration per bout.

\section{Discussion}

The present study aimed to examine sex differences in human physical activity after stroke. The results showed that women had significantly greater Total and LPA walking durations. In contrast, there were no significant sex differences in any parameter in MVPA.

This study showed that women had a significantly greater walking duration per day than men did. Previous studies $^{4-6)}$ have reported mixed results in terms of sex differences in PA after stroke. Our results demonstrated sex differences in participant's walking durations after stroke.

This study showed that the greater walking duration observed in women was attributable to an increased walking duration in LPA compared with that of men. This is consistent with previous studies ${ }^{7,8)}$ that reported healthy older woman spent more time in LPA, such as in household activities, than older men. These findings suggest that, compared with men, women spend more time in housework before stroke and continue to be involved in household activities after stroke. Gender roles could explain the sex differences in LPA in people after stroke. 
Table 2. Comparison of walking duration between men and women

\begin{tabular}{cccccc}
\hline & \multicolumn{2}{c}{ Men $(\mathrm{n}=42)$} & \multicolumn{2}{c}{ Women $(\mathrm{n}=20)$} & \multirow{2}{*}{-value } \\
\cline { 2 - 5 } & mean \pm SD & Median $(25 \%-75 \%)$ & mean \pm SD & Median $(25 \%-75 \%)$ & \\
\hline Total (min) & $115 \pm 59$ & $108(70-152)$ & $140 \pm 41$ & $143(110-164)$ & 0.042 \\
LPA (min) & $68 \pm 45$ & $56(40-86)$ & $103 \pm 36$ & $106(78-128)$ & 0.001 \\
MVPA (min) & $47 \pm 31$ & $45(18-68)$ & $37 \pm 19$ & $34(22-54)$ & 0.45 \\
Short-bout & $30 \pm 22$ & $24(14-43)$ & $26 \pm 14$ & $24(17-31)$ & 1.00 \\
Long-bout & $17 \pm 20$ & $6(0-31)$ & $11 \pm 16$ & $3(0-17)$ & 0.21 \\
\hline
\end{tabular}

Short-bout: lasting 1-9 min, Long-bout: lasting $\geq 10 \mathrm{~min}$

(A) Median duration per bout

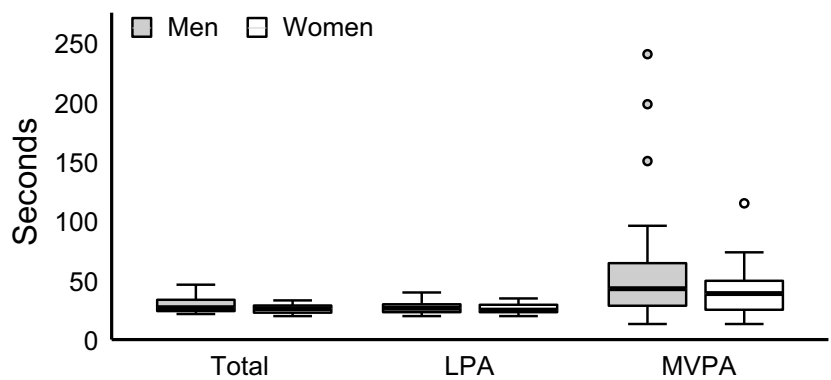

(B) Number of bouts per day

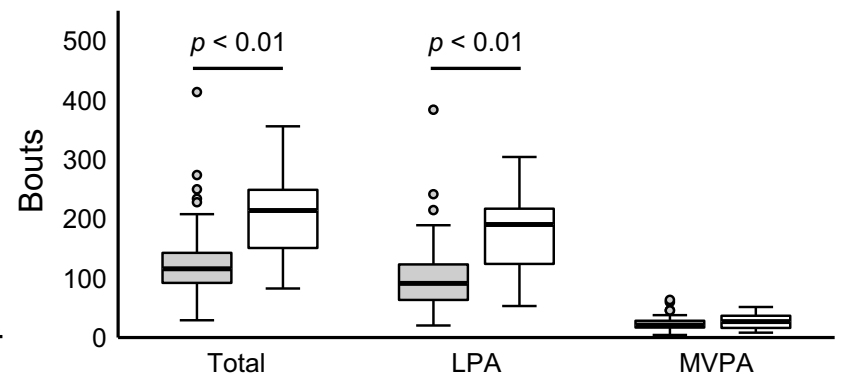

Fig. 1. Comparison of median duration per bout and the number of bouts per day between men and women

The median duration in LPA was $56 \mathrm{~min} /$ day in men and $106 \mathrm{~min} /$ day in women. A previous study ${ }^{23)}$ reported that replacing one hour of sedentary time with LPA was associated with $18 \%$ lower mortality. Thus, differences in LPA duration of approximately 40 minutes between men and women in our sample may affect health outcomes. This study also showed that the greater duration of LPA in women than men was due, not to an increase of duration per bout, but to an increase in walking bouts (Fig. 1). These findings suggest that after stroke, men could benefit from interventions, such as counseling aimed to encourage replacing sedentary time with frequent LPA.

Little is currently known about sex differences in MVPA after stroke. Our results revealed no significant sex differences in MVPA duration, regardless of the length of the walking bout. In this study, $75 \%$ of the total MVPA were short-bout MVPA. This result suggests that short-bout MVPA is easily acceptable for people after stroke compared to long-bout MVPA. Total MVPA duration was strongly associated with lower mortality, regardless of how the MVPA was accumulated ${ }^{24)}$.

We defined the intensity of activity based on the relative indicator of cadence. The advantages of this method are that it is adaptable to a range of stroke participants with various walking abilities. The threshold between LPA and MVPA was 0.655 in the peak cadence ratio, corresponding to a cadence of $69 \pm 9$ steps/min. This cut-off definition was higher than that reported in previous studies ${ }^{25,26)}$, which defined MVPA as $\geq 30$ steps/min. In addition, our cut-off definition was close to the established threshold value of 100 steps/min for MVPA in healthy adults ${ }^{27)}$. Although there is no established definition of MVPA after stroke, we believe that our definition of activity intensity was reasonable and useful for people after stroke with various walking disabilities.

The present study involved several limitations. Firstly, we asked participants to wear a step activity monitor for three consecutive days. However, data for 10 participants were only available for two days. A previous study reported that monitoring for more than a 3-day period is recommended for high reliability ${ }^{28)}$. Secondly, we collected step data every 5 seconds to ensure accurate representation of continuous stepping. Although the optimal epoch length to measure PA for people after stroke remains unclear, our results might have differed if we had collected step data using a longer epoch ${ }^{29)}$. Thirdly, although one female participant walked in a pool during the measurement period, swimming activities were not considered due to restrictions of the step activity monitor. Finally, the results have limited generalizability because our patients had mild walking disability (2-minute walk test of $151 \mathrm{~m}$ and a fastest gait speed of $1.5 \mathrm{~m} / \mathrm{s}$ ).

\section{Conclusion}

In conclusion, sex differences were found in the walking duration in people after stroke. Women more frequently engaged in and spent more time in LPA than men. In con- 
trast, no sex differences were found in MVPA. Our results will be useful for planning interventions to increase PA and decrease sedentary behavior after stroke.

Acknowledgments: This work was supported by JSPS KAKENHI [Grant Number JP17K13065].

Conflict of Interest: The authors declare that there is no conflict of interest.

\section{References}

1) Deijle IA, Van Schaik SM, et al.: Lifestyle Interventions to Prevent Cardiovascular Events After Stroke and Transient Ischemic Attack: Systematic Review and Meta-Analysis. Stroke. 2017; 48: 174-179.

2) Billinger SA, Arena R, et al.: Physical activity and exercise recommendations for stroke survivors: a statement for healthcare professionals from the American Heart Association/American Stroke Association. Stroke. 2014; 45: 2532-2553.

3) Fini NA, Holland AE, et al.: How Physically Active Are People Following Stroke? Systematic Review and Quantitative Synthesis. Physical therapy. 2017; 97: 707-717.

4) English C, Manns PJ, et al.: Physical activity and sedentary behaviors in people with stroke living in the community: a systematic review. Phys Ther. 2014; 94: 185-196.

5) Field M, Gebruers N, et al.: Physical activity after stroke: A systematic review and meta-analysis. ISRN Stroke. 2013; 2013: 113.

6) Thilarajah S, Mentiplay BF, et al.: Factors Associated With Post-Stroke Physical Activity: A Systematic Review and MetaAnalysis. Arch Phys Med Rehabil. 2018; 99: 1876-1889.

7) Azevedo MR, Araújo CL, et al.: Gender differences in leisuretime physical activity. Int J Public Health. 2007; 52: 8-15.

8) Sun F, Norman IJ, et al.: Physical activity in older people: a systematic review. BMC public health. 2013; 13: 449.

9) Amagasa S, Fukushima N, et al.: Light and sporadic physical activity overlooked by current guidelines makes older women more active than older men. Int J Behav Nutr Phys Act. 2017; 14: 59.

10) Butland RJ, Pang J, et al.: Two-, six-, and 12-minute walking tests in respiratory disease. Br Med J (Clin Res Ed). 1985; 11: 1411-1413.

11) Collen FM, Wade DT, et al.: Mobility after stroke: reliability of measures of impairment and disability. Int Disabil Studies. 1990; 12: 6-9.

12) Podsiadlo D and Richardson S: The timed "Up \& Go": a test of basic functional mobility for frail elderly persons. J Am Geriatr Soc. 1991; 39: 142-148.
13) Folstein MF, Folstein SE, et al.: "Mini-mental state". A practical method for grading the cognitive state of patients for the clinician. J Psychiatr Res. 1975; 12: 189-198.

14) Kroenke K, Spitzer RL, et al.: The PHQ-9: validity of a brief depression severity measure. J Gen Intern Med. 2001; 16: 606-613.

15) Krupp LB, LaRocca NG, et al.: The fatigue severity scale. Application to patients with multiple sclerosis and systemic lupus erythematosus. Arch Neurol. 1989; 46: 1121-1123.

16) Hill KD, Schwarz JA, et al.: Fear of falling revisited. Arch Phys Med Rehabil. 1996; 77: 1025-1029.

17) Mudge S, Stott NS, et al.: Criterion validity of the StepWatch Activity Monitor as a measure of walking activity in patients after stroke. Arch Phys Med Rehabil. 2007; 88: 1710-1715.

18) Nakano W, Ohashi Y, et al: : Walking activity in communitydwelling stroke survivors within 1 month after discharge from a rehabilitation setting. Disabil Rehabil. 2020; 42: 1087-1092.

19) Roos MA, Rudolph KS, et al.: The structure of walking activity in people after stroke compared with older adults without disability: a cross-sectional study. Phys Ther. 2012; 92: 1141-1147.

20) Kramer S, Johnson L, et al.: Energy Expenditure and Cost During Walking After Stroke: A Systematic Review. Arch Phys Med Rehabil. 2016; 97: 619-632.e1.

21) Orendurff MS, Segal AD, et al:: The kinematics and kinetics of turning: limb asymmetries associated with walking a circular path. Gait Posture. 2006; 23: 106-111.

22) Orendurff MS, Schoen JA, et al.: How humans walk: bout duration, steps per bout, and rest duration. J Rehabil Res Dev. 2008; 45: 1077-1089.

23) Matthews CE, Keadle SK, et al.: Accelerometer-measured doseresponse for physical activity, sedentary time, and mortality in US adults. Am J Clin Nutr. 2016; 104: 1424-1432.

24) Saint-Maurice PF and Troiano RP: Moderate-to-Vigorous Physical Activity and All-Cause Mortality: Do Bouts Matter? J Am Heart Assoc. 2018; 22: e007678.

25) Manns PJ and Baldwin E: Ambulatory activity of stroke survivors: measurement options for dose, intensity, and variability of activity. Stroke. 2009; 40: 864-867.

26) Michael K and Macko RF: Ambulatory activity intensity profiles, fitness, and fatigue in chronic stroke. Top Stroke Rehabil. 2007; 14: 5-12.

27) Tudor-Locke $C$ and Rowe DA: Using cadence to study freeliving ambulatory behaviour. Sports Med. 2012; 42: 381-398.

28) Mudge S and Stott NS: Test--retest reliability of the StepWatch Activity Monitor outputs in individuals with chronic stroke. Clin Rehabil. 2008; 22: 871-877.

29) Ayabe M, Kumahara H, et al.: Epoch length and the physical activity bout analysis: an accelerometry research issue. BMC research notes. 2013; 6: 20. 